Journal of

Women's Health and Gynecology

\title{
Role of Vaginal DHA on Uterine Contractions in Patients at Low Risk for Preterm Birth
}

\section{Locci Mariavittoria*, Nazzaro Giovanni, Iazzetta Raffaella, Palmieri Teresa, Miranda Marilena, Tirone Monica, Salzano Emilia, De Placido Giuseppe}

Department of Neuroscience, Reproductive Sciences and Odontostomatology; Section of Obstetrics and Gynecology, University Federico II, Naples, Italy

${ }^{\star}$ Corresponding author: Locci Mariavittoria, Department of Neuroscience, Reproductive Sciences and Odontostomatology; Section of Obstetrics and Gynecology, University Federico II, Naples, Italy, Tel: +39337865220, E-mail: mariavittorialocci@virgilio.it

Received Date: May 30, 2016; Accepted Date: August 31, 2016; Published Date: September 02, 2016

Citation: Locci Mariavittoria et al. (2016) Role of Vaginal DHA on Uterine Contractions in Patients at Low Risk for Preterm Birth. J Womens Health Gyn 1: 1-9.

\begin{abstract}
Summary
Preterm labor is a public health problem and to this day, it's the great challenge of modern obstetrics. We conducted a randomized double-blind controlled study. At the admission, all patients were submitted to cardiotocography. Patients with regular contractions but without decreasing of cervical length or funneling at transvaginal sonography were admitted. 90 patients at 24-34 weeks of gestation were finally recruited. Patients were randomly divided in two groups: A group (45 patients) received a single transvaginal dose of docosahexaenoic acid ( 1 gr of DHA); B group (45 patients) received placebo. At 120 minutes, cardiotocographic examination was performed in all patients. A group showed a significant decrease in number and intensity of contractions $(\mathrm{p}<0.05)$ as compared to $\mathrm{B}$ group. The results of our study show that vaginally administered DHA during pregnancy is effective on uterine contractions providing a significant decrease of spontaneous contractions and probably reducing the risk of preterm delivery.
\end{abstract}

Keywords: Preterm delivery; Singleton pregnancies; Uterine contraction; Vaginal DHA

\section{Introduction}

Preterm labor is defined as regular uterine contractions resulting in changes in the cervix that start between 24 weeks of pregnancy and 37 weeks of pregnancy. The preterm birth is the leading cause of perinatal mortality and morbidity, accounting for $28 \%$ of neonatal mortality worldwide. Although the cause is often unknown, a variety of factors may play a role in preterm birth; inflammation is the most common and most important. Vaginal infections seem to play an important role. Infections result in the activation of the inflammatory cascade, which precedes and causes the shortening of the cervix. On this basis, we focused our studies on inflammatory mediators, including cytokines and chemokines, in women's cervix [2]. The fatty acids are precursors of molecules such as prostaglandins, leukotrienes and thromboxanes: all these

(C)2016 The Authors. Published by the JScholar under the terms of the Creative Commons Attribution License http://creativecommons.org/licenses/ by/3.0/, which permits unrestricted use, provided the original author and source are credited. inflammatory mediators play a role in the pathophysiology of preterm birth [7]. The human body can produce the saturated and monounsaturated fatty acids but cannot synthesize omega- 3 and omega- 6 polyunsaturated fatty acids (PUFA). The precursors of these two families- alpha linolenic acid (ALA) and linoleic acid (LA)- are called "essential"; LA and ALA are required in the diet $[3,4]$. Foods with a higher content of omega- fatty acids include salmon, mackerel, sardines, herring, tuna, anchovies (blue fish). Dietary LA serves as the precursor for the $\mathrm{n}-6$ series of polyunsaturated fatty acids (PUFAs) and dietary ALA is the precursor for the n-3 PUFA series. ALA is converted to eicosapentaenoic acid (EPA) and then in docosahexaenoic acid (DHA), while LA is converted to arachidonic acid (AA). DHA is an essential component of cell membranes, where it modulates, together with the EPA, the right degree of fluidity and permeability. 
The optimal omega-3/omega- 6 fatty acids ratio is $1: 5$ whereas the western diet ranges from 10:1 to $25: 1$, fueling concern that this diet, characterized by low fish consumption, leads to a decline of omega-3 PUFAs intake in favour of omega-6 PUFAs and to an altered omega-6/omega-3 ratio from 1:10 to $1: 20$, a so called pro-inflammatory ratio. In fact, omega- 3 fatty acids are essential and can only be obtained from the diet [8]. The increased intake of omega-3 in proportion to other fats would result in the down-regulation of the synthesis of omega- 6 and consequently the decreased production of pro-inflammatory cytokines [7].

The American Dietetic Association (ADA) with the Dietitians of Canada [2] recommend at least $500 \mathrm{mg} /$ day of PUFAs for all healthy adults including pregnant and lactating women. The European Commission with the International Society for the Study of Fatty Acids and Lipids (ISSFAL) specifically recommends that pregnant and lactating women consume a minimum of $200 \mathrm{mg}$ DHA per day [7,8]. These recommendations could be met by consuming 1 to 2 portions per week of fish with high content of omega-3 fatty acids, which is the recommendation by Health Canada [8] and the United States Dietary Guidelines Advisory Committee for all women [4]. Since this considerations we evaluate the role of docosahexaenoic acid on uterine contractions to show if this molecule can be of use in reducing the early symptoms and signs of preterm delivery.

\section{Materials and Methods}

We conducted a randomized double-blind controlled study by February 2014 to November 2014. The study was conducted at the Reproductive Medicine Unit of the University of Naples Federico II. We enrolled 90 symptomatic patients between 2434 week of gestation, for threatened preterm labour. The study was approved by the Ethics Committee of the University Federico II (Protocol's number 268/13). The patients underwent informed consent. The patients were divided into 2 groups according to random criteria, 45 patients in the treatment group (A group) and 45 to the placebo group (B group). 140 pregnant patients with pelvic pain at 24-34 weeks were evaluated. At the admission patients were submitted to digital vaginal examination, transvaginal ultrasonography and cardiotocography (CTG). Only patients with regular contractions at CTG (4 in 20 minutes or 8 in 60 minutes, lasting at least 30 seconds) (1) and without clinical or sonographic modifications of the cervix were considered for the study.

The inclusion criteria were the following:

1. Caucasian women between 18 and 38 years of age

2. Singleton pregnancy

3. Uterine contractions reported

4. Regular uterine activity demonstrated to cardiotocography pattern ( $>4$ contractions in 20 minutes)

5. Cervical length $>25 \mathrm{~mm}$ and absence of funneling at transvaginal ultrasound

6. Intact membranes

7. Obstetric history negative for preterm labor.
Patients with the following characteristics were excluded:

1. Maternal age $<18$ and $>38$

2. Multiple pregnancy

3. Cervical length $<25 \mathrm{~mm}$ and / or presence of funneling at transvaginal ultrasound

4. Infections of the genito-urinary tract (positive vaginal/urine culture)

5. Intra-amniotic infections, based on clinical and biochemical parameters: maternal temperature $>38^{\circ} \mathrm{C}$, malodorous vaginal discharge, maternal leucocytosis ( $>15000 \mathrm{cell} / \mathrm{mm} 3)$, maternal tachycardia ( $>100$ beats $/ \mathrm{min})$, uterine tenderness

6. Pre-pregnancy or gestation pathologies (such as maternal autoimmune diseases, antiphospholipid syndrome, gestational hypertension, preeclampsia)

Finally, 90 patients were enrolled in the study. Patients were randomly allocated in the 2 group, the treatment group (A group) and the placebo group (B group) using computer-generated numbers in sealed envelopes (Figure 1).

The patients were randomized to receive a single vaginal capsule of docosahexaenoic acid (-Metra-medical device CE Class III produced by Pharmarte, registration number $\mathrm{CE}$ 0373 containing fish oil rich in DHA - 1g) or vaginal placebo capsule. The placebo capsules were specially manufactured to look identical to the DHA capsules. The capsules were placed in sacs and then stored in envelopes numbered from 1 to 90 . The envelopes were numbered and randomized according to computer-generated randomization tables to ensure an equal number of patients in each arm. Access to the randomization code was available only to the pharmacist who manufactured the placebo and packed the envelops and was not available to any of the treating physicians or patients. Patients were revalued by cardiotocography pattern after 120 minutes after administration of the preparation.

The primary endpoint was to determine the number of uterine contractions ( $<4$ in 20 minutes or $<8$ in 60 minutes) by tocography after 120 minutes from vaginal administration of one gram of DHA.

\section{Statistical analysis}

Statistical analysis was performed using Graph Pad Prism 4.0 software (Graph Pad Software Inc., La Jolla, CA). The t test was used for assessing the significance of the difference between continuous variables. The X2 test or the Fisher exact test was used to assess the statistical significance of categorical varables. Differences associated with $\mathrm{p}$ values lower than 0.05 were considered statistically significant. The results are reported as mean \pm standard deviation (SD). A significant difference was found in terms of number of uterine contractions at CTG between the two groups $(\mathrm{p}<0.05)$. 


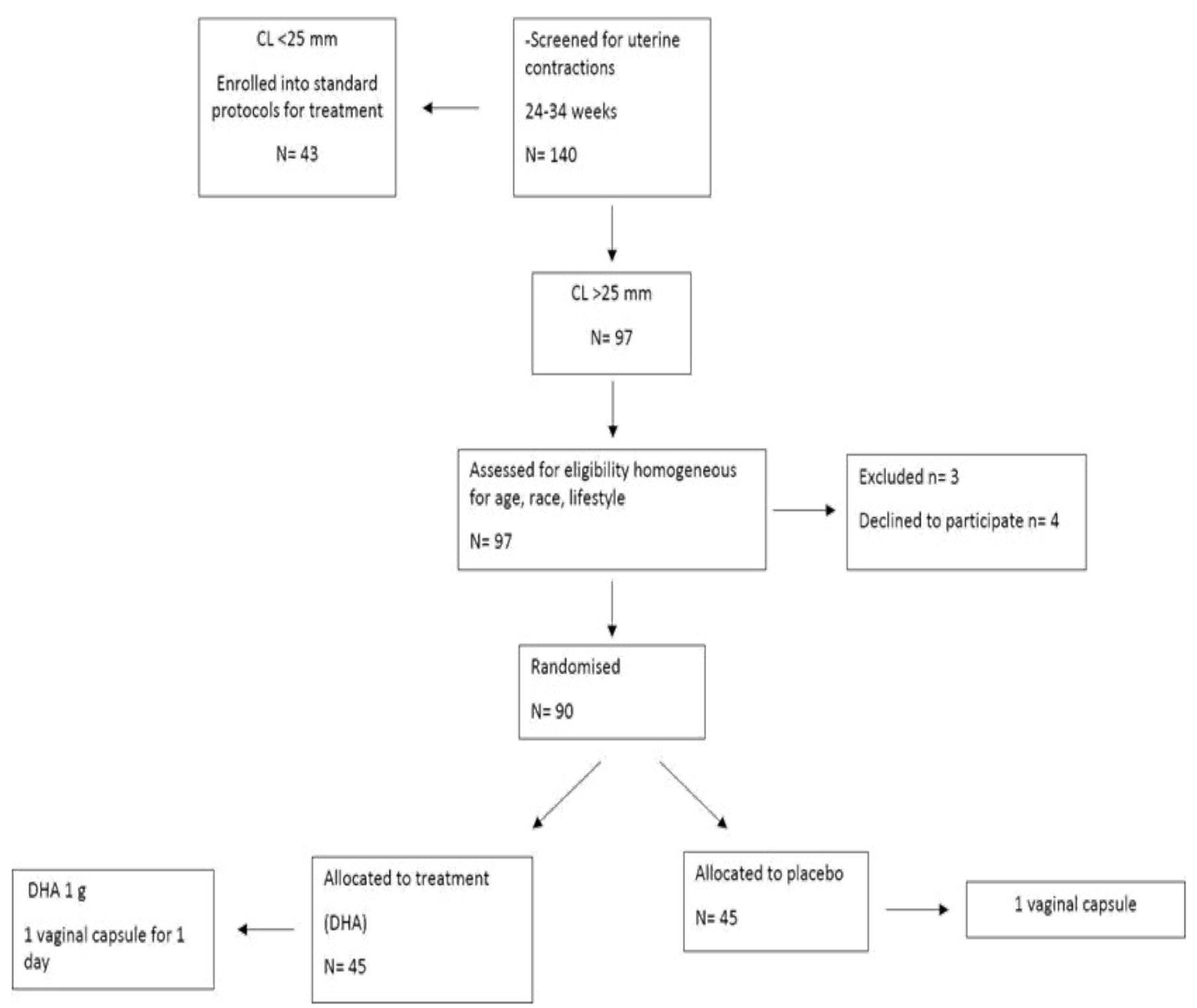

Figure 1: Flow chart for the allocation of the patients.

\section{Results}

In A group the mean number of contractions was significantly lower after the treatment $(6.11 \pm 1.30$ vs $3.13 \pm 3.33, \mathrm{p}=0.0001)$ (Figure 2) while in B group the difference was not significant $(5.28 \pm 1.32$ vs $5.55 \pm 1.45, \mathrm{p}=0.36)$ (Figure 3). Patients in A group showed a significant reduction in the mean number of contractions at cardiotocography after 120 minutes of docosahexaenoic acid somministration than in B group ( $3.13 \pm 3.33$ vs $5.55 \pm 1.45$, $\mathrm{p}=0.0001$ ) (Figure 4). The number of patients with a reduction of uterine contractions was significantly higher in A group. In particular, in A group, 20 patients (44\%) had no contractions at the post-treatment CTG, 11 patients (24\%) had a reduction in the number of contractions ( $<6$ in 60 minutes); in 9 patients $(20 \%)$ the number of contractions was unchanged and in 5 patients (11\%) was detected worsening of symptoms. In B group, no patients showed improvement of symptoms, 28 patients (62\%) presented an unchanged cardiotocographic pattern before and after the placebo, 15 patients (33\%) showed worsening of the symptomatology and only 2 patients (5\%) had a reduction in the number of contractions (Table 1).

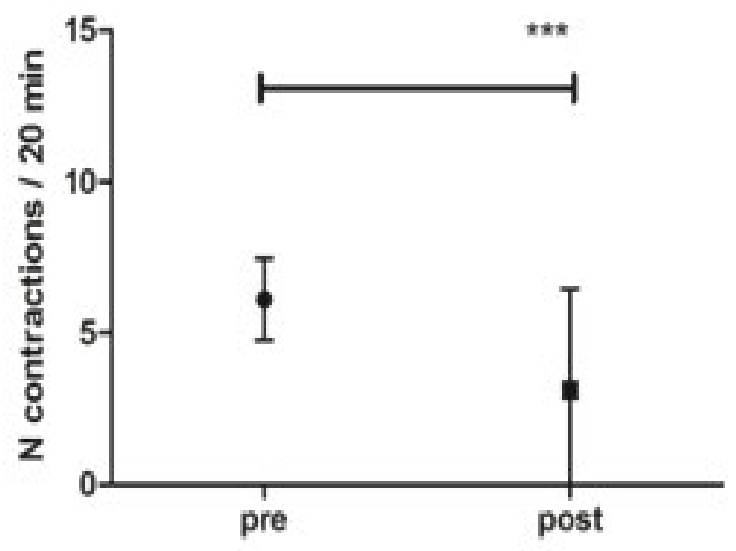

Figure 2: Mean number of contractions before and after treatment with DHA (A group).

${ }^{\star * *}$ p 0.0001 


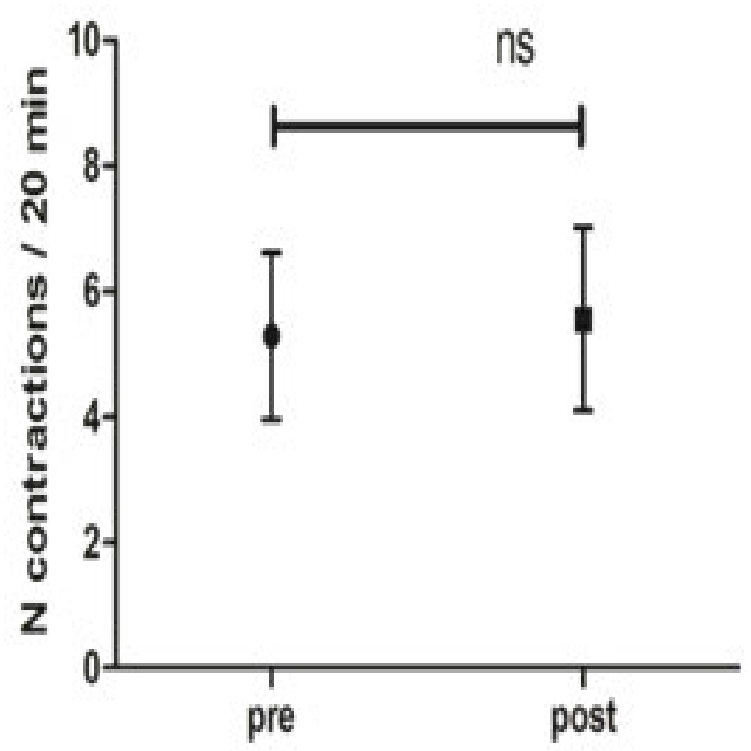

Figure 3: Mean number of contractions before and after placebo (B group).

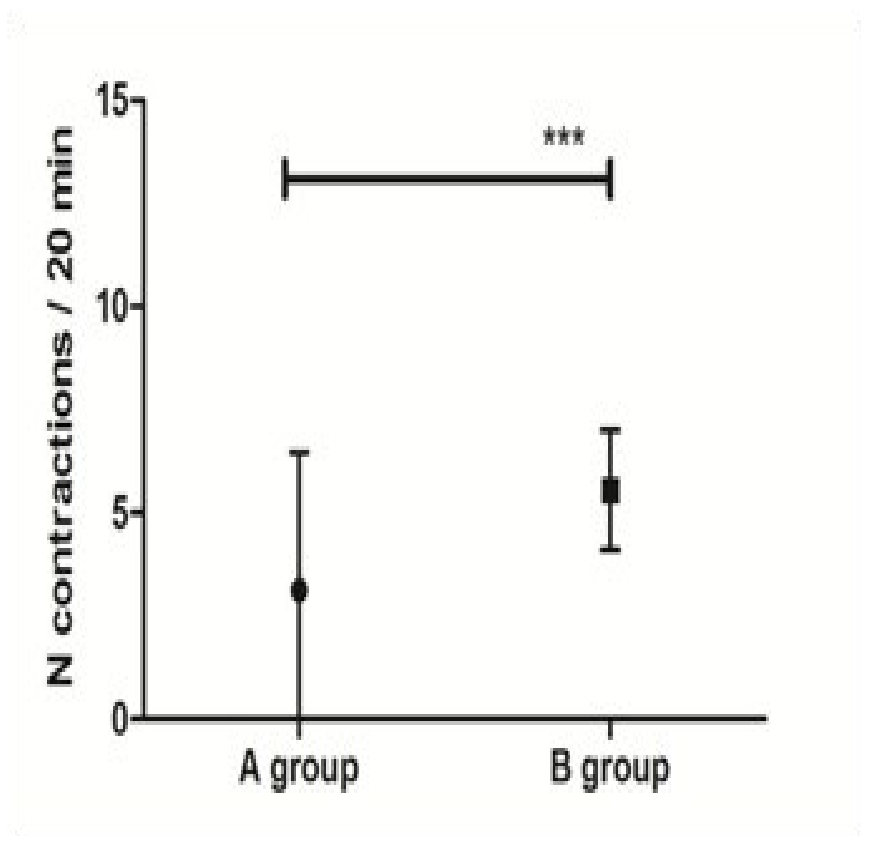

Figure 4: Mean number of contractions after treatment in A group e B group. ${ }_{* * *}$ p 0.0001

Table 1: Effect of treatment on uterine contractions.

\begin{tabular}{|l|l|l|l|l|}
\hline $\begin{array}{l}\text { Frequency of uter- } \\
\text { ine contractions }\end{array}$ & No contractions & Decreasing & Unchanged & Increasing \\
\hline A group $n=45$ & $20(44)$ & $11(24)$ & $9(20)$ & $5(11)$ \\
\hline B group $n=45$ & $0(0)$ & $2(5)$ & $28(62)$ & $15(33)$ \\
\hline P value & $<0.001$ & $<0.05$ & $<0.001$ & $<0.05$ \\
\hline
\end{tabular}

Data are presented as number of cases and percentage (\%).

\section{Discussion}

Variation of dietary intake plays a key role in reducing some risks associated with pregnancy, such as risk of fetal and infant mortality, low birth weight $[12,13]$ and premature births [2]. In this study, we observed that vaginally administered DHA during pregnancy acts on uterine contractions, in symptomatic women resulting in muscle relaxation and induces disappearance of spontaneous contractions in many cases. Several randomized controlled trials have shown that supplementation with omega-3 may influence the birth process by delaying the onset of labor, reducing the risk of recurrent preterm birth and in animal studies it seems to have a tocolytic effect $[2,15]$. The exact DHA's mechanisms of action are not well understood, the eicosanoid-mediated changes on myometrial contractions and the connective tissue remodeling [5,12,15] seem involved. A high omega-6/omega-3 fatty acid ratio will result in increasing proinflammatory eicosanoid production; these metabolites have been associated with labor at term and preterm.
A higher intake of omega 3 fatty acids leads to a down-regulation of the synthesis of omega- 6 fatty acids and consequently reduces the production of prostaglandins responsible for cervical changes in early labor. In additional, several studies have shown that DHA significantly reduces the secretion of IL-8 and IL-6 from the amnion [2,7]. These cytokines are strongly associated with the onset of labor and this activity may contribute to the ability of DHA, introduced by diet supplements, to prolong gestation and to decrease the risk of preterm delivery before 34 weeks. Animals with n- 6 fatty acid deficiency and those fed with high dose of n-3 fatty acids have depressed prostaglandins synthesis and increased length of gestation [8]. Another possible effect on the duration of pregnancy could be linked to the disorganization of the electrical myometrial activity: this could determine a delay in the onset of rhythmic and regular myometrial contractions [4]. 
Several randomized controlled trials showed a reduction of preterm delivery with DHA supplementation during pregnancy [6] $n-3$ supplementation was associated, compared to no supplementation in control subjects, with significantly greater duration of pregnancy [8]. The supplementation with marine oil in pregnancy is associated with a mean gestational age at delivery 2.6 days longer than women allocated placebo or no treatment and did have a lower risk of giving birth before 34 completed weeks' gestation compared with placebo [7].

Studies performed until today are based on the effects of oral administration of $200 \mathrm{mg}$ omega-3 fatty acids, however, since oral administration is subject to hepatic metabolism and rapid inactivation, we hypothesized that the use of a vaginal formulation of DHA may provide a better bioavailability due to uterine-first-pass-effect, bypassing liver metabolism and directly reaching the cervix. Therefore, we evaluated the efficacy of intravaginal formulation of 1 gr of DHA, which, in fact, resulted in a more effective activity on myometrium than the oral one. Avoiding the first-pass effect, vaginal administration could have a more potent cervical action, with a reduction of omega 6 synthesis and consequent lower production of PGE2 and PGF2a.

\section{Conclusion}

Maternal long-chain polyunsaturated fatty acids status, particularly docosahexaenoic acid (DHA), during pregnancy may influence maternal and infant outcomes [2,13]. Maternal lifestyle and nutrition have long been recognized as important factors for both perinatal health and for the long-term health of the infant. Fish oil capsules are nearly devoid of mercury and other harmful compounds and can serve to augment omega-3 fatty acids in the diet. The implications of DHA supplementation on fetal development, maternal outcomes and later infant growth is worth being elucidated and is promising in its potential for a positive impact on fetal and maternal outcomes [6]. The vaginal administration allows the use of dosage five times higher than the oral one, similar to the dosage tested in animal studies [3]. Furthermore, by passing the gastrointestinal absorption, DHA acts directly on the cervix and on the feto placental unit, contrasting hyperoxia [2]. Placental hyperoxia contributes to a persistent imbalance between pro-inflammatory and anti-inflammatory mechanisms that leads to preterm birth and neonatal injuries [17]. The potential benefits of DHA intake ( $1 \mathrm{gr}$ ) in patients at risk of preterm birth should be confirmed and further studies are needed to evaluate the effects and effectiveness of long-term treatment with DHA.

\section{References}

1) AAP \& ACOG (1997) Guidelines for Perinatal Care. (4th edn) 100. Livello VI (ACOG III).

2) Baguma-Nibasheka M, Brenna JT, Nathanielsz PW (1999) Delay of preterm delivery in sheep by omega-3 long-chain polyunsaturates. Biol Reprod 60: 698-701.

3) Churc MW, Jen KL, Dowhan LM, Adams BR, Hotra JW ( 2008) Excess and deficient omega-3 fatty acid during pregnancy and lactation cause impaired neural transmission in rat pups. Neurotoxicol Teratol 30: 107-117.

4) Dietary Guidelines for Americans (2005) Report of the Dietary Guidelines Advisory Committee on Dietary Guidelines for Americans.

5) Giorlandino C, Giannarelli D (2013) Effect of vaginally administered DHA fatty acids on pregnancy outcome in high risk pregnancies for preterm delivery: a double blinded randomised controlled trial. Journal of Prenatal Medicine 7: 42-45.

6) Hanebutt FL, Demmelmair H, Schiessl B, Larqué E, Koletzko B (2008) Long-chain polyunsaturated fatty acid (LC-PUFA) transfer across the placenta. Clin Nutr 27: 685-693.

7) Hansen HS, Olsen SF (1988) Dietary (n-3)-fatty acids, prostaglandins, and prolonged gestation in humans. Prog Clin Biol Res 282: 305-317.

8) Health Canada (2002) Prenatal Nutrition. Health Canada, Ottawa, Ont, Canada.

9) Holmar NT (1998) The slow discovery of the importance of omega 3 essential fatty acids in human health. J Nutr. 128: 427S-433S.

10) Hornstra G (2000) Essential fatty acids in mothers and their neonates. Am J Clin Nutr 71:1262S-1269S.

11) Koletzko, B., Cetin, I., and Brenna, J.T (2007) Dietary fat intakes for pregnant and lactating women. Br. J. Nutr. 98: 873-877.

12) Koletzko B, Lien E, Agostoni C, Böhles H, Campoy C, et al. (2008) The roles of long-chain polyunsaturated fatty acids in pregnancy, lactation and infancy: review of current knowledge and consensus recommendations. J. Perinat. Med 36: 5-14.

13) Kris-Etherton PM, Innis S (2007) American Dietetic Association, Dietitians of Canada. Position of the American Dietetic Association and Dietitians of Canada: dietary fatty acids. J. Am. Diet. Assoc. 107: 1599-1611.

14) Locci M, Nazzaro G, Miranda M, Salzano E, Montagnani S, et al. (2013) Vaginal lactoferrin in asymptomatic patients at low risk for pre-term labour for shortened cervix: cervical length and interleukin-6 changes. J Obstet Gynaecol. 33: 144-148.

15) Makrides M, Duley L, Olsen SF (2006) Marine oil, and other prostaglandin precursor, supplementation for pregnancy uncomplicated by pre-eclampsia or intrauterine growth restriction. Cochrane Database Syst Rev. 19.

16) Olsen SF, Hansen HS, Sorensen TI, et al. (1986) Intake of marine fat, rich in (n-3)-polyunsaturated fatty acids, may increase birthweight by prolonging gestation. Lancet 2:367-369.

17) Sharma D, Nkembi AS, Aubry E, Houeijeh A (2015) Maternal PUFA $\omega-3$ Supplementation Prevents Neonatal Lung Injuries Induced by Hyperoxia in Newborn Rats. Int J Mol Sci 14: 22081-22093.

18) Szajewska H, Horvath A, Koletzko B (2006) Effect of n-3 long chain polyunsaturated fatty acid supplementation of women with low-risk pregnancies on pregnancy outcomes and growth measures at birth: a meta-analysis of randomized controlled trials. Am J Clin Nutr 83: 1337-1344. 
Submit your manuscript to a JScholar journal and benefit from:

a Convenient online submission

๑ Rigorous peer review

I Immediate publication on acceptance

- Open access: articles freely available online

- High visibility within the field

q Better discount for your subsequent articles

Submit your manuscript at http://www.jscholaronline.org/submit-manuscript.php 